\title{
Cystatin $B$ as a potential diagnostic biomarker in ovarian clear cell carcinoma
}

\author{
AKANE TAKAYA $^{1,2}$, WEI-XIA PENG ${ }^{1}$, KOUSUKE ISHINO $^{1}$, MITSUHIRO KUDO $^{1}$, \\ TETSUSHI YAMAMOTO ${ }^{3}$, RYUICHI WADA ${ }^{1}$, TOSHIYUKI TAKESHITA ${ }^{2}$ and ZENYA NAITO $^{1}$ \\ ${ }^{1}$ Department of Integrated Diagnostic Pathology, Nippon Medical School, Tokyo 113-8602; ${ }^{2}$ Division of Reproductive \\ Medicine, Perinatology and Gynecologic Oncology, Graduate School of Medicine, Nippon Medical School, \\ Tokyo 113-8603; ${ }^{3}$ School of Pharmacy, Kinki University, Osaka 577-8502, Japan
}

Received November 17, 2014; Accepted December 29, 2014

DOI: $10.3892 /$ ijo.2015.2858

\begin{abstract}
Epithelial ovarian cancer (EOC) consists of four major subtypes: clear cell carcinoma (CCC), endometrioid adenocarcinoma (EA), mucinous adenocarcinoma (MA) and serous adenocarcinoma (SA). Relative to the other subtypes, the prognosis of $\mathrm{CCC}$ is poor due to a high recurrence rate and chemotherapy resistance, but $\mathrm{CCC}$-specific biomarkers have yet to be identified. With the aim of identifying diagnostic and treatment biomarkers for $\mathrm{CCC}$, we analyzed 96 cases of EOC (32 CCC, 13 EA, 19 MA, 32 SA) using liquid chromatography/mass spectrometry (LC/MS) followed by immunohistochemistry (IHC) and quantitative reverse transcription PCR (RT-qPCR). Semi-quantification of protein differences between subtypes showed upregulation of 150 proteins and downregulation of 30 proteins in CCC relative to the other subtypes. Based on hierarchical clustering that revealed a marked distinction in the expression levels of cystatin B (CYTB) and Annexin A4 (ANXA4) in CCC relative to the other subtypes, we focused the study on CYTB and ANXA4 expression in EOCs by IHC, RT-qPCR and western blot analyses using tissue specimens and cultured cells. As a result, compared to the other subtypes, CCC showed significantly high expression levels of CYTB and ANXA4 in the analyses. To examine the possibility of CYTB and ANXA4 as serum diagnostic biomarkers of $\mathrm{CCC}$, we checked the protein
\end{abstract}

Correspondence to: Professor Zenya Naito, Department of Integrated Diagnostic Pathology, Nippon Medical School, 1-1-5 Sendagi, Bunkyo-ku, Tokyo 113-8602, Japan

E-mail: naito@nms.ac.jp

Abbreviations: EOC, epithelial ovarian cancer; CCC, clear cell carcinoma; EA, endometrioid adenocarcinoma; MA, mucinous adenocarcinoma; SA, serous adenocarcinoma; CYTB, cystatin B; ANXA4, Annexin A4; FFPE, formalin-fixed paraffin-embedded; IHC, immunohistochemistry

Key words: epithelial ovarian cancer, shotgun proteomics, formalinfixed paraffin-embedded tissue, clear cell carcinoma, cystatin B, Annexin A4 levels in conditioned media and cell lysates using culture cells. Compared with the other subtypes, CCC cell lines showed a significantly higher level of expression of CYTB in both conditioned media and cell lysates, while ANXA4 showed a higher level of expression in cell lysates only. Our results demonstrate that CYTB and ANXA4 overexpression may be related to carcinogenesis and histopathological differentiation of CCC. CYTB may be a secreted protein, and may serve as a potential serum diagnostic biomarker of CCC, while ANXA4 may be useful as an intracellular marker.

\section{Introduction}

Epithelial ovarian cancer (EOC) is the leading cause of gynecological cancer-related deaths worldwide because it is generally detected at a late, incurable stage. While the serum CA125 level is commonly used for detection of ovarian cancers, the sensitivity and specificity of this parameter are questionable. In clear cell carcinoma (CCC), for example, half of all cases do not show any increase in CA125. In addition, non-cancerous diseases such as endometriosis, adenomyosis and pelvic inflammation may also result in elevated CA125 (1). Despite the fact that the four histological subtypes of EOC [CCC, endometrioid adenocarcinoma (EA), mucinous adenocarcinoma (MA), serous adenocarcinoma (SA)] differ from each other with regard to precursor lesion, accumulated course of genetic alterations, and chemosensitivity, current clinical treatments for all four subtypes are nearly the same $(2,3)$. Relative to other subtypes, the prognosis of CCC is poor due to a high recurrence rate and chemotherapy resistance; nonetheless, CCC-specific biomarkers have yet to be identified (4).

Shotgun proteomics is a method of identifying and profiling proteins from complex mixtures using a combination of liquid chromatography (LC) and mass spectrometry (MS) (5-13). Recent technological developments have made it possible to extract mixtures of peptides from formalin-fixed paraffin-embedded (FFPE) tissues, including cancers such as EOC, for proteome analysis (14-17). In this study, we performed global shotgun proteome analysis on FFPE tissues derived from EOCs, and focused on the CCC subtype in an effort to identify novel candidate proteins for early diagnosis as well as new therapeutic targets. 


\section{Materials and methods}

Ovarian cancer tissue specimens. FFPE tissues from 96 patients with ovarian cancer who underwent surgery at Nippon Medical School Hospital (Tokyo, Japan) and Chiba-Hokuso Hospital of Nippon Medical School (Chiba, Japan) between 2005 and 2012 were analyzed in this study (Table I). Subtypes included 32 cases of CCC, 13 of EA, 19 of MA and 32 of SA. Two investigators (A. Takaya and W-X Peng) reassessed the histological type according to the World Health Organization (WHO) classification (2014), and clinicopathological stage according to the 2012 International Federation of Gynecology and Obstetrics (FIGO) staging of EOC. This study was performed in accordance with the principles of the Revised Declaration of Helsinki, 2013, and informed consent was acquired from all patients for the use of specimens.

Cell lines. Human ovarian cancer cell lines (JHOC-5 and -9, JHOM-1, JHOS-2 cells) $(18,19)$ were obtained from RIKEN BioResource Center (Ibaraki, Japan). JHOC-5 (RBRCRCB1520) and JHOC-9 (RBRC-RCB2226) was derived from human CCC. JHOM-1 (RBRC-RCB1676) was derived from a human MA, while JHOS-2 (RBRC-RCB1521) was derived from a human SA.

Cells were cultivated in a medium consisting of Dulbecco's modified Eagle's medium (DMEM): Nutrient Mixture F-12 (HamF-12) at 1:1 (Gibco, Grand island, NY, USA) medium containing $10 \%$ heat-inactivated fetal bovine serum (FBS) and $0.1 \mathrm{mM}$ MEM non-essential amino acids (NEAA) (Gibco) at $37^{\circ} \mathrm{C}$ under a humidified $5 \% \mathrm{CO}_{2}$ atmosphere. All lines were tested over the course of $\sim 3$ passages.

Conditionedmedium (CM) and cell lysates from humanovarian

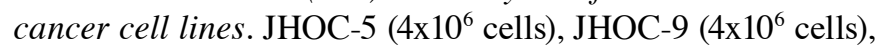

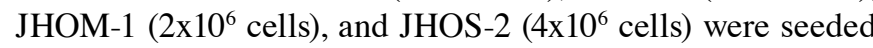
into $150-\mathrm{cm}^{2}$ flasks. After $48 \mathrm{~h}$, culture medium was replaced with $30 \mathrm{ml}$ serum-free medium, and cells were cultivated for additional $24 \mathrm{~h}$. Each culture was then collected and centrifuged at $400 \mathrm{x} \mathrm{g}$ for $20 \mathrm{~min}$ at $4^{\circ} \mathrm{C}$, and supernatants were separated and concentrated at $4^{\circ} \mathrm{C}$ by centrifugation at 14,000 x g using Amicon Ultra 3K filters (Millipore, Billerica, MA, USA). The supernatants were collected as conditioned media, protein concentrations were measured using the Pierce $660 \mathrm{~nm}$ Protein Assay kit (Thermo Fisher Scientific, Inc., Rockford, IL, USA), and conditioned media were stored at $-80^{\circ} \mathrm{C}$. Human ovarian cancer cell lines $\left(5 \times 10^{5}\right.$ cells $/ 15 \mathrm{ml} / 100 \mathrm{~mm}$ dish) were also seeded and cultured for $72 \mathrm{~h}$ in 1:1 DMEM:HamF12 medium containing $10 \%$ heat-inactivated FBS. After cultivation, cells were lysed in buffer containing $2 \mathrm{M}$ thiourea (Nacalai Tesque, Inc., Kyoto, Japan), 7 M urea (Wako Pure Chemical Industries, Ltd., Tokyo, Japan), 3\% 3-[(3-cholamidoproryl) dimethylammonio]-1-propanesulfonate (Dojindo, Kumamoto, Japan), and 1\% Triton X-100 (Sigma-Aldrich, St. Louis, MO, USA), followed by treatment with $10 \%$ trichloroacetic acid (TCA) for $30 \mathrm{~min}$. Cell lysates were centrifuged for $30 \mathrm{~min}$ at $20,400 \mathrm{x} \mathrm{g}$ at $4^{\circ} \mathrm{C}$ to remove precipitated TCA (Wako Pure Chemical Industries, Ltd.) and cellular debris, and supernatants were collected as cell extract. Protein concentrations were measured using Pierce $660 \mathrm{~nm}$ Protein Assay kit and cleared lysates were stored at $-80^{\circ} \mathrm{C}(20)$.
Table I. Summary of EOC cases studied.

\begin{tabular}{|c|c|c|c|c|}
\hline & CCC (32) & EA (13) & MA (19) & SA (32) \\
\hline Age (range, years) & $37-71$ & $35-72$ & $23-83$ & $30-76$ \\
\hline $\begin{array}{l}\text { Mean age at } \\
\text { diagnosis (years) }\end{array}$ & 54 & 52 & 59 & 55 \\
\hline $\mathrm{T} 1$ & 19 & 10 & 14 & 3 \\
\hline $\mathrm{T} 2$ & 5 & 1 & 0 & 2 \\
\hline $\mathrm{T} 3$ & 6 & 1 & 3 & 18 \\
\hline $\mathrm{T} 4$ & 2 & 1 & 2 & 9 \\
\hline NO & 28 & 13 & 19 & 15 \\
\hline N1 & 4 & 0 & 0 & 17 \\
\hline M0 & 30 & 12 & 17 & 23 \\
\hline M1 & 2 & 1 & 2 & 9 \\
\hline \multicolumn{5}{|l|}{ FIGO } \\
\hline I & 19 & 10 & 14 & 3 \\
\hline II & 5 & 1 & 0 & 2 \\
\hline III & 6 & 1 & 3 & 16 \\
\hline IV & 2 & 1 & 2 & 11 \\
\hline \multicolumn{5}{|l|}{$\begin{array}{l}\text { Recurrence } \\
\text { after treatment }\end{array}$} \\
\hline$(-)$ & 24 & 12 & 18 & 17 \\
\hline$(+)$ & 8 & 1 & 1 & 15 \\
\hline
\end{tabular}

EOC, epithelial ovarian cancer; CCC, clear cell carcinoma; EA, endometrioid adenocarcinoma; MA, mucinous adenocarcinoma; SA, serous adenocarcinoma; FIGO, International Federation of Gynecology and Obstetrics.

Microdissection of FFPE EOC tissue. FFPE tissues originating from $36 \mathrm{EOC}$ patients consisted of $9 \mathrm{CCC}, 8 \mathrm{EA}$, $7 \mathrm{MA}$, and $12 \mathrm{SA}$. EOC patients were used for proteomic analysis. Ten-micrometer sections were deparaffinized with xylene and rehydrated through a series of graded alcohols (100, 90, 80 and 70\%). After staining with Mayer's Hematoxylin for $5 \mathrm{~min}$, cancerous regions were dissected manually with the aid of a Nikon MultiZoom AZ100M microscope (Nikon Instech Co., Ltd., Tokyo, Japan). Some cancers with abundant desmoplastic stroma were dissected away from stromal regions by Laser Microdissection (LMD) using a Leica LMD6000 microscope (Leica Camera AG, Solms, Germany).

Protein idetification by LC-MS/MS analysis. Proteins were extracted from the FFPE tissue sections with lysis buffer [6 M guanidine hydrochloride, $40 \mathrm{mM}$ Tris- $\mathrm{HCl}, \mathrm{pH} 8.2,65 \mathrm{mM}$ dithiothreitol (DTT) (Wako Pure Chemical Industries, Ltd.)] according to a previous report (12) and protein concentrations were measured using the Bradford method. Trypsin digestion of extracted proteins was performed as described by Bluemlein and Ralser (21) with slight modifications. In brief, $10 \mu \mathrm{g}$ of 
extract from each specimen were first reduced in $45 \mathrm{mM}$ DTT and $20 \mathrm{mM}$ Tris[2-carboxyethyl]phosphine (TCEP) for $30 \mathrm{~min}$ at $37^{\circ} \mathrm{C}$, and then alkylated in $100 \mathrm{mM}$ iodoacetamide (IAA) (both from Wako Pure Chemical Industries, Ltd.) for $30 \mathrm{~min}$ at $37^{\circ} \mathrm{C}$ in the dark. Following digestion with trypsin (Agilent Technologies, Inc., Santa Clara, CA, USA) at $37^{\circ} \mathrm{C}$ for $24 \mathrm{~h}$, each sample was desalted using PepClean C-18 Spin Columns (Thermo Fisher Scientific, Inc.) according to the manufacturer's instructions.

Samples consisting of $\sim 2 \mu \mathrm{g}$ purified peptides were injected into a peptide L-trap column (Chemicals Evaluation and Research Institute, Tokyo, Japan) using an HTS PAL Autosampler (CTC Analytics AG, Zwingen, Switzerland) and then further separated with Advance-nano UHPLC on a reverse-phase $\mathrm{C} 18$ column (Zaplous column $\alpha, 3-\mu \mathrm{m}$ diameter gel particles and $100 \AA$ pore size, $0.1 \times 150 \mathrm{~mm}$ ) (both from AMR Inc., Tokyo, Japan). The mobile phases consisted of solution A ( $0.1 \%$ formic acid in water) and solution B $(0.1 \%$ formic acid in acetonitrile). The column was developed at a flow rate of $500 \mathrm{ml} / \mathrm{min}$ with a $5-35 \% \mathrm{~B}$ gradient over the course of $120 \mathrm{~min}$. Peptides were analyzed using an amaZon ETD ion trap mass spectrometer (Bruker Daltonics, Billerica, MA, USA).

All MS/MS spectral data were compared to the Swiss-Prot Homo sapiens database using Mascot (version 2.3.01; Matrix Science, London, UK) to identify the proteins. The search criteria were: trypsin enzyme, allowance of up to two missed cleavage peptides, mass tolerance $\pm 0.5 \mathrm{Da}, \mathrm{MS} / \mathrm{MS}$ tolerance \pm 0.5 Da, fixed modifications of cysteine carbamidomethylation, variable modifications of methionine oxidation.

Spectral counting analysis of identified proteins. To compare protein expression patterns among tissue samples in the shotgun analysis, we used the spectral counting method. The number of peptide spectra identified with high confidence (significance, $\mathrm{p}<0.05$ ) served as the spectral count value. Relative amounts of identified proteins in each sample were obtained using the normalized spectral abundance factor (NSAF) (22), allowing for identification of 47 candidate proteins with NSAF values $>0.01$.

Data processing and cluster analysis. The NSAF values of candidate proteins were clustered hierarchically using Cluster 3.0 (23) and visualized with Java TreeView (24).

Immunohistochemical analysis. FFPE tissue sections $(3 \mu \mathrm{m})$ were immunostained for cystatin B (CYTB) (EPR3931; Abcam, Tokyo, Japan) and Annexin A4 (ANXA4) (HPA007393; Atlas Copco, Stockholm, Sweden) using the Histofine Simple Stain MAX-PO (R) kit (Nichirei Corp., Tokyo, Japan). Sections were pre-treated in an autoclave at $121^{\circ} \mathrm{C}$ for $15 \mathrm{~min}$ in $10 \mathrm{mM}$ citrate buffer (pH 6.0 for CYTB and $\mathrm{pH} 9.0$ for ANXA4) for retrieval of the antigen. After blocking endogenous peroxidase activity with $0.3 \% \mathrm{H}_{2} \mathrm{O}_{2}$ in methanol, anti-CYTB antibody (1:300 in dilution) or anti-ANXA4 antibody (1:300 in dilution) was applied, and the slides were incubated for $16 \mathrm{~h}$ at $4^{\circ} \mathrm{C}$. Bound antibodies were detected using Histofine Simple Stain MAX-PO (R) or (M) reagents (Nichirei Corp.). Mayer's Hematoxylin was used for counterstaining.
Two investigators (Takaya A. and Peng W-X) conducted blind evaluations of each section. Sections with tumor cells were scored on staining intensity ( 0 , no stain; 1 , weak; 2 , moderate; 3 , strong) and estimated percentage of tumor cells $(0,0-5 \% ; 1,6-30 \% ; 2,31-60 \% ; 3,61-100 \%)$. IHC scores were the sum of intensity and percentage scores; cases with IHC>5 formed the high expression group, and all others the low expression group.

$R T-q P C R$ analysis. Total RNA was extracted from each FFPE EOC tissue sample using the RNeasy FFPE kit (Qiagen, Valencia, CA, USA), and from JHOC-5 and -9, JHOM-1, JHOS-2 cells using the NucleoSpin RNA kit (U0955B; Takara Bio, Inc., Osaka, Japan) according to manufacturer's instructions. cDNAs were synthesized using a SuperScript VILO cDNA Synthesis kit (11754-050; Invitrogen Life Technologies, Carlsbad, CA, USA). Expression levels of CYTB or ANXA4 mRNAs were estimated from quantitative reverse transcription PCR (RT-qPCR) reactions with target and control cDNAs using the TaqMan Universal PCR Master Mix, no AmpErase ${ }^{\circledR}$ UNG (4324018) (Applied Biosystems, Alameda, CA, USA), and primers and TaqMan probes for CYTB (Hs00164368_s1) and ANXA4 (Hs00984874_s1) and 18S rRNA (Hs03928990_g1) (Applied Biosystems). The optimized program involved denaturation at $95^{\circ} \mathrm{C}$ for $10 \mathrm{~min}$, followed by 45 cycles of amplification at $95^{\circ} \mathrm{C}$ for $15 \mathrm{sec}$ and $60^{\circ} \mathrm{C}$ for $1 \mathrm{~min}$. RT-qPCR results are expressed as an internal standard concentration ratio, target/18S rRNA, and analyzed using the $\Delta \Delta \mathrm{Ct}$ method. mRNA expression was measured in triplicate (25-27).

Western blot analysis. Equivalent protein samples were resolved using 6 or $13 \%$ sodium dodecyl sulfate-polyacrylamide gel electrophoresis (SDS-PAGE), and proteins were then transferred electrophoretically onto polyvinylidene fluoride (PVDF) membrane (Millipore, Tokyo, Japan). After blocking with $5 \%$ skim milk in Tris-buffered saline consisting of $0.2 \mathrm{M}$ Tris- $\mathrm{HCl}, 150 \mathrm{mM} \mathrm{NaCl}$, and $0.05 \%$ Tween-20 (TBS-T) for $1 \mathrm{~h}$ at room temperature, membranes were incubated with anti-CYTB antibody (dilution, 1:1,000), anti-ANXA4 antibody (dilution, 1:200), or anti- $\beta$-actin antibody (dilution, 1:10,000) for $2 \mathrm{~h}$ at room temperature. After a 30-min wash in TBS, blots were incubated with HRP-conjugated anti-rabbit IgG antibody (dilution, 1:8,000) or HRP-conjugated anti-mouse IgG antibody (dilution, 1:8,000) for $1 \mathrm{~h}$ at room temperature. Immunoreactive products were visualized using SuperSignal West Dura Chemiluminescence Substrate (Thermo Fisher Scientific, Inc.) and quantified using Chemi Doc XRS System and Quantity One Software (Bio-Rad Laboratories, Inc., Tokyo, Japan). Three independent blots of each tissue sample were evaluated.

Statistical analysis. The data are shown as mean \pm SEM. Two groups of CCC and non-CCC were compared using Mann-Whitney U test or Fisher's test. Significant differences in expression levels between EOC subtypes were assessed using one-way ANOVA Tukey's multiple comparisons. All statistical analyses were performed using GraphPad Prism version 5 (Graphpad Software, Inc., La Jolla, CA, USA), with $\mathrm{p}<0.05$ considered significant in each analysis. 


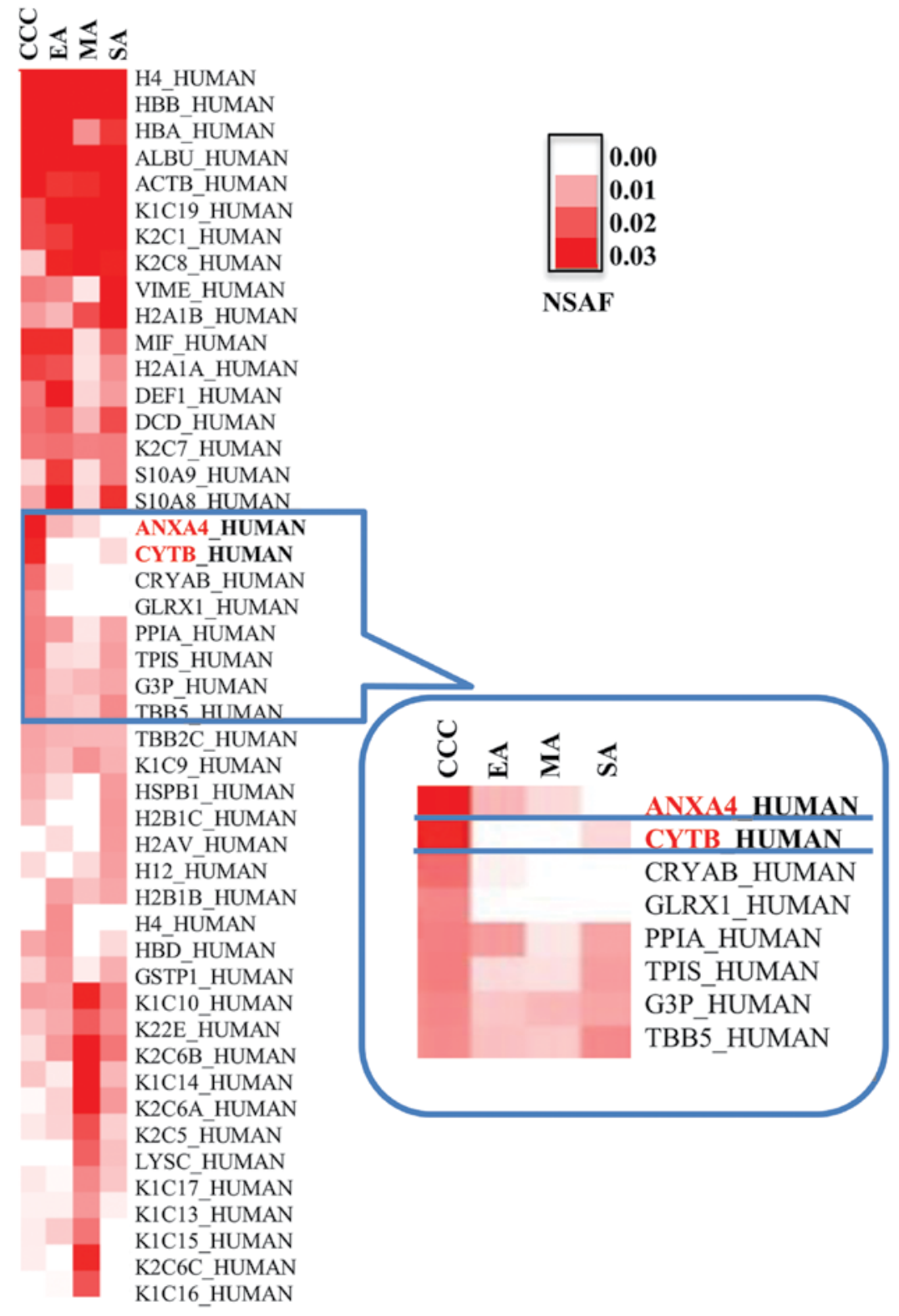

Figure 1. Cluster heat map. Hierarchical clustering of candidate proteins from a semi-quantitative differential expression analysis of epithelial ovarian cancer (EOC) formalin-fixed paraffin-embedded (FFPE) tissues. A heat map was made from the result of hierarchical cluster analysis of 47 selected proteins with normalized spectral abundance factor (NSAF) value of $>0.01$ using Cluster 3.0 and Java TreeView softwares. UniProtKB/Swiss-Prot protein identifiers of candidate proteins are indicated on the right side of the map.

\section{Results}

Protein identification and comparison of expression levels between CCC and other EOC subtypes. Shotgun proteome analysis of the four EOC subtypes identified 399 different proteins in the FFPE tissues, of which 197 were found in CCC, 179 in EA, 141 in MA, and 178 in SA, respectively. Among the 399 proteins, 67 were common to all EOCs. Unique to one subtype were 60 proteins from CCC, 62 from EA, 35 from $\mathrm{MA}$, and 36 from SA.

To identify CCC-specific biomarker candidates, we carried out cluster analyses using proteomic results and visualized results with a heat map. Hierarchical clustering revealed a clear distinction in the expression of CYTB and ANXA4 in CCC relative to other EOC subtypes (Fig. 1), leading us to focus on CYTB and ANXA4 as potential diagnostic markers of CCC.
IHC analysis of CYTB and ANXA4. Spectral measurements of CYTB and ANXA4 expression levels were confirmed by immunohistochemical analysis of FFPE from CCC, EA, MA and SA cases ( $\mathrm{n}=32,13,19$ and 32, respectively). CCC cell sections showed strong CYTB and ANXA4 expression in the cell membranes, cytoplasm and nucleus (Fig. 2); in contrast, normal epithelial cells are only weak or negative for cytoplasmic staining of CYTB and ANXA4. High expression of CYTB was observed in $50 \%$ of CCC, which was significantly more than that in EA (31\%), MA (11\%) or SA (13\%) subtypes ( $<<0.001$; Fisher's test) (Table II). Among the 16 CCC cases that showed strong CYTB expression, 11 cases were in early stage (FIGO stage I and II) and five cases were in late stage (FIGO stage III and IV). There were also significantly more cases of high ANXA4 expression in the CCC subtype (94\%) relative to EA (16\%), MA (53\%), or SA (10\%) (p<0.001; Fisher's 


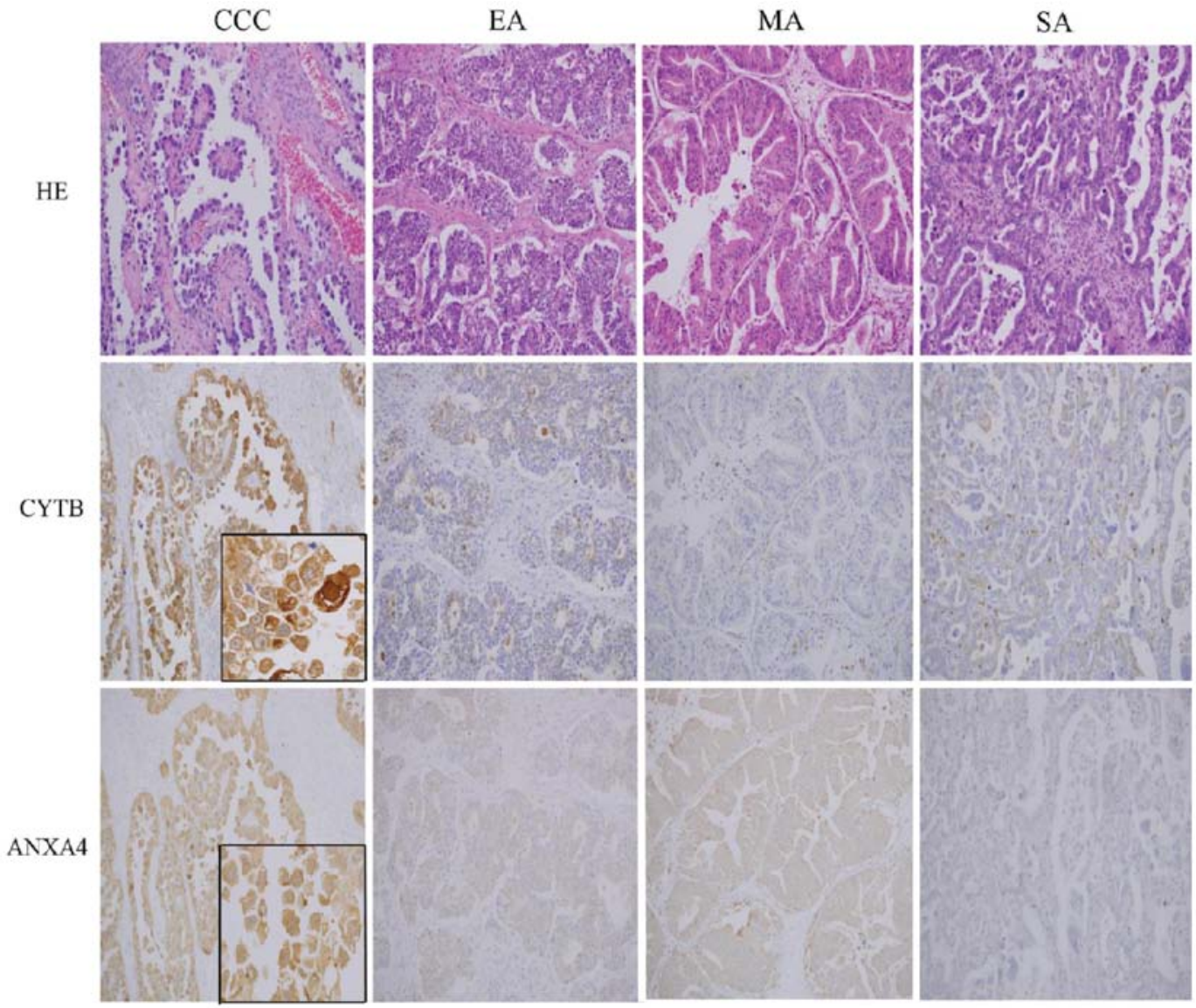

Figure 2. Immunohistochemical staining of cystatin B (CYTB) and Annexin A4 (ANXA4) in four epithelial ovarian cancer (EOC) subtypes. Top row: hematoxylin-stained controls (HE) (x200) from clear cell carcinoma (CCC), endometrioid adenocarcinoma (EA), mucinous adenocarcinoma (MA) and serous adenocarcinoma (SA) sections. Middle and lower rows: CYTB and ANXA4 immunoreactivity, respectively. The lower right sides of CYTB and ANXA4 of CCC demonstrate the images in larger scale (x600 and x400). CYTB and ANXA4 demonstrated differential immunostaining in that CCC sections showed strong CYTB and ANXA4 expression in the cell membranes and cytoplasm, and nucleus relative to other EOC subtypes.

Table II. Immunodetection of CYTB and ANXA4 in four EOC subtypes.

\begin{tabular}{lccrrrr}
\hline & Protein expression & CCC (32) & EA (13) & MA (19) & SA (32) & P-value $^{\text {a }}$ \\
\hline CYTB & High (score 5-6) & $16(50 \%)$ & $4(31 \%)$ & $2(11 \%)$ & $4(13 \%)$ & $<0.001$ \\
& Low (score 0-4) & $16(50 \%)$ & $9(69 \%)$ & $17(89 \%)$ & $28(87 \%)$ & \\
\multirow{3}{*}{ ANXA4 } & High (score 5-6) & $30(94 \%)$ & $2(16 \%)$ & $10(53 \%)$ & $3(10 \%)$ & $<0.001$ \\
& Low (score 0-4) & $2(6 \%)$ & $11(84 \%)$ & $9(47 \%)$ & $29(90 \%)$ & \\
\hline
\end{tabular}

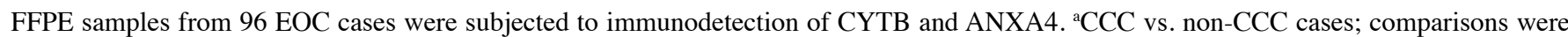
performed using Fisher's test. CYTB, cystatin B; ANXA4, Annexin A4; EOC, epithelial ovarian cancer; CCC, clear cell carcinoma; EA, endometrioid adenocarcinoma; MA, mucinous adenocarcinoma; SA, serous adenocarcinoma; FFPE, formalin-fixed paraffin-embedded.

test). With regard to stage, $100 \%$ of late stage cases and $91 \%$ of cases of early stage CCC showed high ANXA4 expression.

RT-qPCR analysis in FFPE tissues and EOC cell lines. Following manual or LMD dissection of EOC regions, we performed RT-qPCR analysis of isolated tissues with CYTB, ANXA4, and 18S mRNA specific primers. In EOC FFPE tissues, normalized expression level of CYTB mRNA (Fig. 3A) was significantly higher in CCC than in non-CCC subtypes $(\mathrm{p}<0.0001$, Mann-Whitney U test). Between individual subtypes, CYTB mRNA expression in CCC was significantly higher than that in MA or SA ( $p<0.05$, Tukey's test). The significant difference was not found in CYTB mRNA expression level between CCC and EA. ANXA4 mRNA (Fig. 3B) expression level among EOC FFPE tissues was significantly higher in CCC than in non-CCC subtypes $(\mathrm{p}<0.0001$, Mann-Whitney U test), and the level of ANXA4 was also higher in CCC than in any one of the other subtypes alone $(\mathrm{p}<0.05$, Tukey's test). Moreover, among EOC cell lines, the expression levels of CYTB (Fig. 3C) and ANXA4 (Fig. 3D) mRNAs in 
A

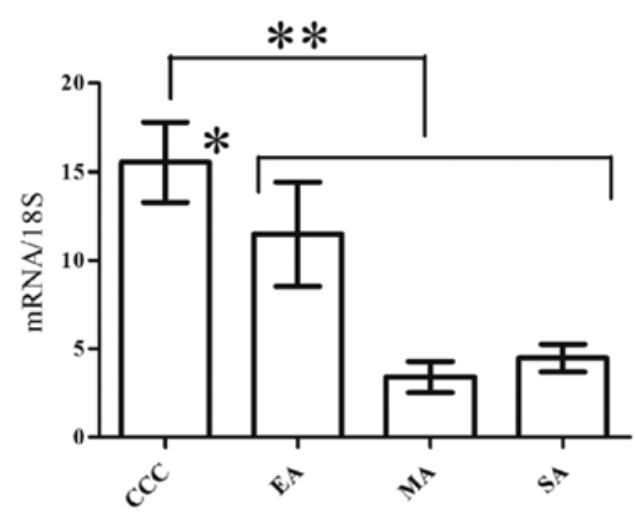

$\mathrm{C}$

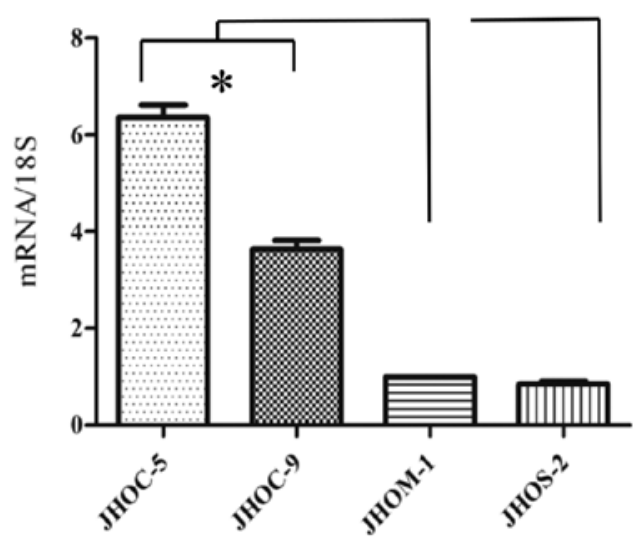

B

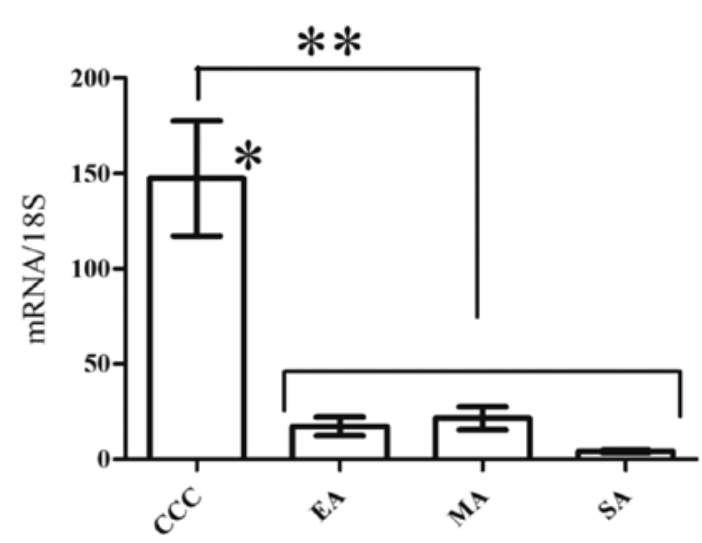

$\mathrm{D}$

ANXA4

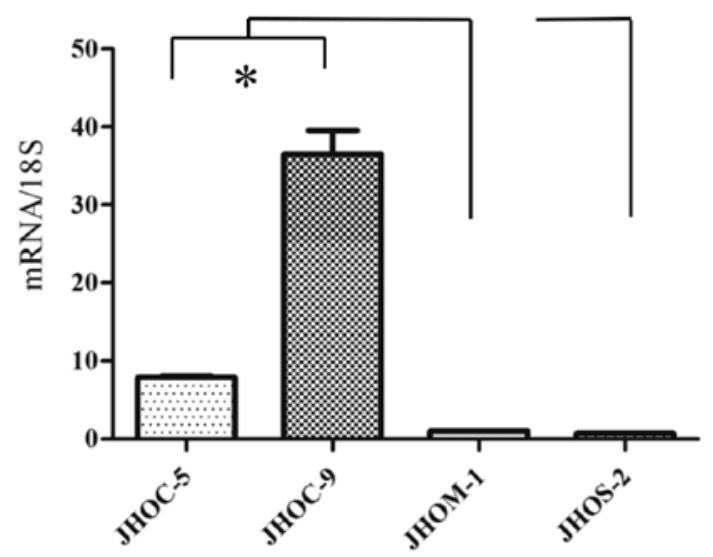

Figure 3. Quantitative reverse transcription PCR (RT-qPCR) of cystatin B (CYTB) and Annexin A4 (ANXA4). Expression levels (y-axis) are depicted as the ratio of expression of CYTB or ANXA4 mRNAs relative to ribosomal 18S mRNAs as measured using quantitative PCR. (A) In epithelial ovarian cancer (EOC) formalin-fixed paraffin-embedded (FFPE) tissues, expression of CYTB mRNA was significantly higher in clear cell carcinoma (CCC) than in non-CCC subtypes (** $\mathrm{p}<0.0001$, Mann-Whitney U test). Between individual subtypes, CYTB mRNA expression in CCC was significantly higher than that in mucinous adenocarcinoma (MA) or serous adenocarcinoma (SA) ("p<0.05, Tukey's test). (B) In EOC FFPE tissues, ANXA4 mRNA expression level was significantly higher in CCC than in non-CCC subtypes ( $*$ p $<0.0001$, Mann-Whitney U test), and the level of ANXA4 was also higher in CCC than in any one of the other subtypes alone ("p<0.05, Tukey's test). In EOC cell lines, the expression levels of (C) CYTB and (D) ANXA4 mRNAs were significantly higher in JHOC-5 and -9 than in each of JHOM-1 and JHOS-2 ("p<0.05, Tukey's test). All data are shown as mean \pm SEM.

JHOC-5 and -9 were significantly higher than those in each of JHOM-1 and JHOS-2 ( $\mathrm{p}<0.05$, Tukey's test). CCC cell lines showed significantly higher levels of CYTB and ANXA4 than that of non-CCC cell lines ( $<<0.05$, Mann-Whitney U test).

$C Y T B$ and ANXA4 levels in CM and cell lysate of EOC cell lines. It is known that CYTB is a secretory protein and is released into the circulation in patients with various cancer patients, including bladder cancer (28) and hepatocellular carcinoma (29). To determine the secretion from cells to culture medium, we confirmed the presence of CYTB and ANXA4 in the cell lysate (Fig. 4A) and CM (Fig. 4B) collected from EOC cell lines. In western blot analysis relative amounts of immunoreactive proteins were measured against a $\beta$-actin standard. CYTB protein levels in both conditioned media and cell lysates were significantly higher in JHOC-5 and -9 cell lines than that in each of JHOM-1 and JHOS-2 cell lines $(\mathrm{p}<0.05$, Tukey's test). In addition, upon comparing CYTB protein level in both conditioned media and cell lysates between CCC and non-CCC cell lines, we found that CCC cell lines showed significantly higher level than non-CCC cell lines ( $\mathrm{p}<0.05$, Mann-Whitney U test). ANXA4 protein level in cell lysate in JHOC-5 and -9 cell lines was higher than that in each of JHOM-1 and JHOS-2 cell lines ( $<<0.05$, Tukey's test). However, no ANXA4 protein was observed in CM of JHOC-5, JHOM-1 and JHOS-2 cell lines.

\section{Discussion}

CYTB is a member of the cystatin superfamily, which consists of endogenous inhibitors of lysosomal cysteine proteinases involved in the degradation of connective tissue and basement membrane proteins. Aberrant control of stromal degradation may contribute to the increased tissue proteolysis that allows cancer cells to spread during neoplastic transformation or tumor progression $(28,30,31)$. When compared to matched normal and benign tissue counterparts, colon, lung and gastric tumor tissues display an imbalance in cystatin expression levels (32). In this study, proteome analysis followed by hierarchical clustering analysis, revealed higher expression of 
A
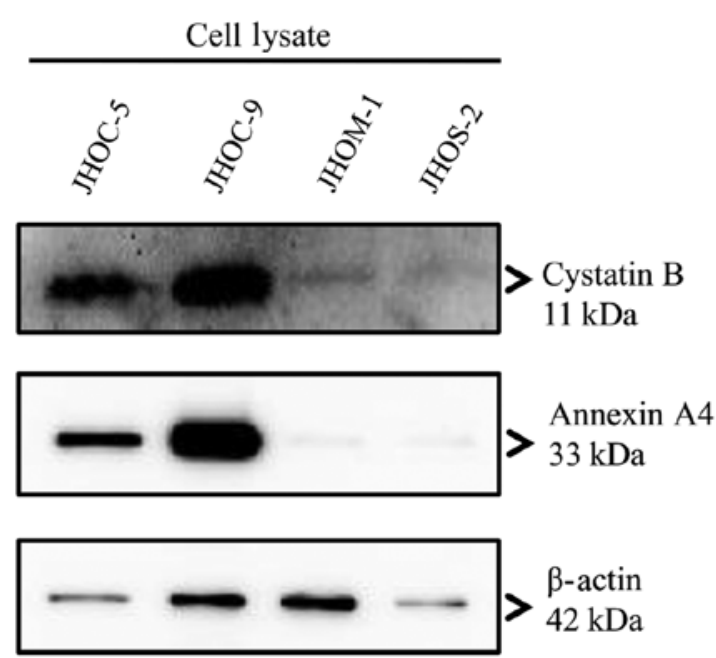

CYTB cell lysate

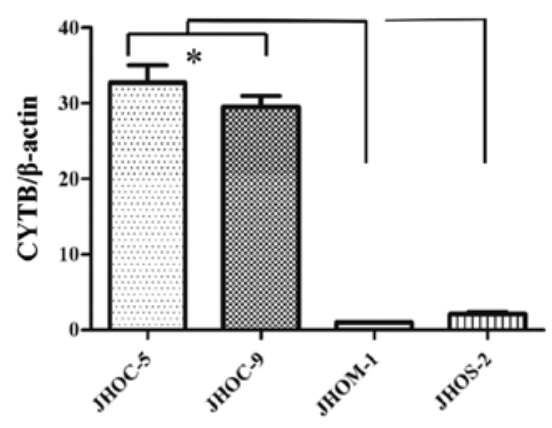

ANXA4 cell lysate

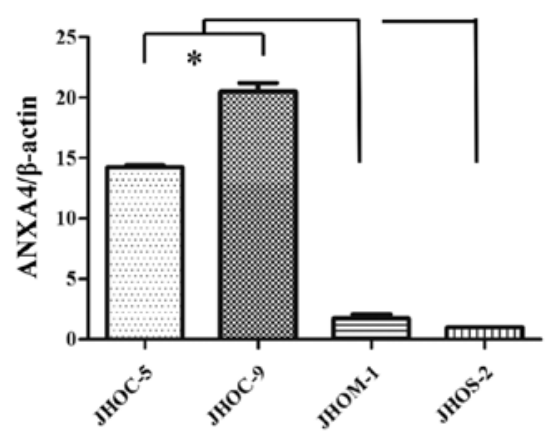

B
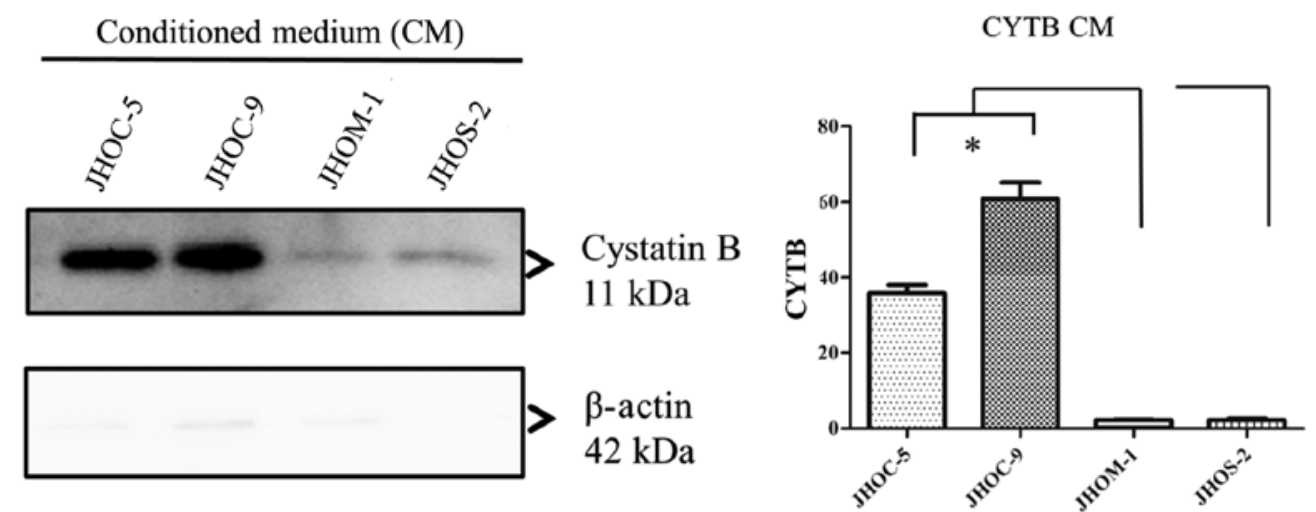

Figure 4. Cystatin B (CYTB) and Annexin A4 (ANXA4) immunoblots of cell lysates and conditioned medium (CM) from four epithelial ovarian cancer (EOC) cell lines. In western blot analysis, relative amounts of immunoreactive proteins were measured against a $\beta$-actin standard. To determine the secretion from cells to culture medium, we confirmed the presence of CYTB and ANXA4 in (A) the cell lysate and (B) the CM collected from EOC cell lines. CYTB protein levels in both conditioned media and cell lysates were significantly higher in JHOC-5 and -9 cell lines than in each of JHOM-1 and JHOS-2 cell lines ( ${ }^{*} \mathrm{p}<0.05$, Tukey's test). ANXA4 protein level in cell lysate of JHOC-5 and -9 cell lines was higher than that in each of JHOM-1 and JHOS-2 cell lines ("p $<0.05$, Tukey's test). However, no ANXA4 protein was observed in CM of JHOC-5, JHOM-1 and JHOS-2 cell lines. All data are shown as mean \pm SEM.

CYTB and ANXA4 in CCC compared with other subtypes. These results were confirmed by the subsequent validating studies using IHC, RT-qPCR and western blot analyses. Moreover, cases in the early stage of CCC also demonstated high expression of CYTB and ANXA4. These results suggest that CYTB and ANXA4 overexpression may be related to carcinogenesis and histopathological differentiation of CCC, repressing CYTB expression may be effective against CCC progression and CYTB may be a potential treatment target for CCC.

One previous study reported CYTB as a serum marker equivalent to $\alpha$-fetoprotein for diagnosis of hepatocellular carcinoma (29). In transitional cell carcinoma, the CYTB level in urine has been reported to correlate positively with tumor grade, stage, and shorter time to disease recurrence and progression (28). The serum CYTB level has also been reported to be higher in patients with ovarian cancer relative to benign ovarian tumor, including high CYTB in ascites fluid (33). To examine the possibility of CYTB as serum diagnostic or treatment biomarker of CCC, we checked the protein level in conditioned media and cell lysates using culture cells. Compared with other subtypes, CCC cell lines showed a significantly higher level of expression of CYTB in both conditioned media and cell lysates. Our results demonstrate that CYTB may be a secreted protein, and CYTB may serve as a potential serum diagnostic or treatment biomarker of CCC. On the other hand, a CCC cell line showed higher expression of ANXA4 protein in cell lysate only compared to non-CCC cell lines. This result suggests that 
ANXA4 may not be a secreted protein, and it may be useful as an intracellular marker.

ANXA4 is a member of the calcium-dependent phospholipid-binding protein family. Our study of ANXA4 expression in FFPE tissues derived from CCC cases agrees with prior reports of high expression in CCC tumors (34-36). These results suggest that ANXA4 may play an important role in CCC carcinogenesis, and also demonstrate that FFPE specimens are appropriate and reliable tissues for proteome analysis. ANXA4 has been shown to play a role in membrane permeability and is involved in modulating drug resistance in cancer cells (36), exo- and endocytosis, as well as fibrinolysis. Like HNF-1 $\beta$, the expression pattern of ANXA4 renders it a molecular signature for cancer pathophysiology $(37,38)$. ANXA4 overexpression is associated with the cell proliferation, chemoresistance, and progression of various carcinomas including ovarian $\mathrm{CCC}$, colorectal carcinoma, gastric cancer and renal carcinoma (39-41). Studies of ANXA4 knock-down cells document significant growth retardation, loss of migration, and greater sensitivity to carboplatin, suggesting that ANXA4 may be involved in CCC chemoresistance $(35,36)$. Our present study may suggest that ANXA4 overexpression may be used for predicting the chemoresistance cases of EOC including CCC. Further studies will be needed to clarify the mechanism between ANXA4 protein expression and the EOC chemoresistance.

In summary, this study provides further evidence supporting the use of CYTB and ANXA4 as diagnostic markers for CCC, as well as for investigation of new clinical therapies that modulate these proteins in order to suppress tumor progression or surmount chemoresistance. The proteomic profiling of four different subtypes of EOC shown here suggests a method for correlating biological targets of disease more directly to histopathological classifications, and may contribute to more individualized treatments for EOC patients in the future.

\section{Acknowledgements}

We would like to thank Mr. Takenori Fujii, Mr. Kiyoshi Teduka, Ms. Yoko Kawamoto, Ms. Kiyoko Kawahara as well as Ms. Taeko Suzuki for technical assistance (Department of Integrated Diagnostic Pathology, Nippon Medical School, Tokyo, Japan). This study was supported in part by Grants-in-aid for Clinical Rebiopsy Bank Project for Comprehensive Cancer Therapy Development to Z.N. from Ministry of Education, Culture, Sport, Science, and Technology (Tokyo, Japan) (MEXT), 2013-2017 (S1311022).

\section{References}

1. du Bois A, Lück HJ, Meier W, et al: A randomized clinical trial of cisplatin/paclitaxel versus carboplatin/paclitaxel as first-line treatment of ovarian cancer. J Natl Cancer Inst 95: 1320-1329, 2003.

2. Kitawaki J, Ishihara $\mathrm{H}$, Koshiba $\mathrm{H}$, et al: Usefulness and limits of CA-125 in diagnosis of endometriosis without associated ovarian endomeriomas. Hum Reprod 20: 1999-2003, 2005.

3. Ozols RF, Bundy BN, Greer BE, et al: Phase III trial of carboplatin and paclitaxel compared with cisplatin and paclitaxel in patients with optimally resected stage III ovarian cancer: A Gynecologic Oncology Group study. J Clin Oncol 21: 3194-3200, 2003.
4. Uekuri C, Shigetomi H, Ono S, Sasaki Y, Matsuura M and Kobayashi H: Toward an understanding of the pathophysiology of clear cell carcinoma of the ovary (Review). Oncol Lett 6: 1163-1173, 2013

5. Old WM, Meyer-Arendt K, Aveline-Wolf L, et al: Comparison of label-free methods for quantifying human proteins by shotgun proteomics. Molecular \& cellular proteomics. Mol Cell Proteomics 4: 1487-1502, 2005.

6. Toyama A, Suzuki A, Shimada T, et al: Proteomic characterization of ovarian cancers identifying annexin-A4, phosphoserine aminotransferase, cellular retinoic acid-binding protein 2, and serpin B5 as histology-specific biomarkers. Cancer Sci 103: 747-755, 2012.

7. Yamamoto T, Kudo M, Peng WX and Naito Z: Analysis of protein expression regulated by lumican in PANC-1 cells using shotgun proteomics. Oncol Rep 30: 1609-1621, 2013.

8. Wang JJ, Liu Y, Zheng Y, Lin F, Cai GF and Yao XQ: Comparative proteomics analysis of colorectal cancer. Asian Pac J Cancer Prev 13: 1663-1666, 2012.

9. Zhu Y, Wu R, Sangha N, et al. Classifications of ovarian cancer tissues by proteomic patterns. Proteomics 6: 5846-5856, 2006.

10. Morgan TM, Seeley EH, Fadare O, Caprioli RM and Clark PE: Imaging the clear cell renal cell carcinoma proteome. J Urol 189: 1097-1103, 2013.

11. Tan Y, Ma SY, Wang FQ, et al: Proteomic-based analysis for identification of potential serum biomarkers in gallbladder cancer. Oncol Rep 26: 853-859, 2011.

12. Jiang X, Jiang X, Feng S, Tian R, Ye M and Zou H: Development of efficient protein extraction methods for shotgun proteome analysis of formalin-fixed tissues. J Proteome Res 6: 1038-1047, 2007.

13. Sprung RW Jr, Brock JW, Tanksley JP, et al: Equivalence of protein inventories obtained from formalin-fixed paraffin-embedded and frozen tissue in multidimensional liquid chromatography-tandem mass spectrometry shotgun proteomic analysis. Mol Cell Proteomics 8: 1988-1998, 2009.

14. Kawamura T, Nomura M, Tojo H, et al: Proteomic analysis of laser-microdissected paraffin-embedded tissues: (1) stage-related protein candidates upon non-metastatic lung adenocarcinoma. J Proteomics 73: 1089-1099, 2010.

15. Bateman NW, Sun M, Bhargava R, et al: Diffetential proteomic analysis of late-stage and recurrent breast cancer from formalin-fixed paraffin-embedded tissues. J Proteome Res 10: 1323-1332, 2011.

16. Naidoo K, Jones R, Dmitrovic B, et al: Proteome of formalin-fixed paraffin-embedded pancreatic ductal adenocarcinoma and lymph node metastases. J Pathol 226: 756-763, 2012.

17. Rodriguez-Rigueiro T, Valladares-Ayerbes M,Haz-Conde M, et al: A novel procedure for protein extraction from formalin-fixed paraffin-embedded tissues. Proteomics 11: 2555-2559, 2011

18. Yamada K, Tachibana T, Hashimoto H, et al: Establishment and characterization of cell lines derived from serous adenocarcinoma (JHOS-2) and clear cell adenocarcinoma (JHOC-5, JHOC-6) of human ovary. Hum Cell 12: 131-138, 1999.

19. Hayashi M, Okuda T, Chiba H, Nagatsuka M and Okai T: The analysis of toll-like receptor signal pathway in human ovarian cancer cells. J Showa Med Assoc 70: 211-221, 2010.

20. Yamamoto T, Matsuda Y, Kawahara K, Naito Z and Ishiwata T: Keratinocyte growth factor stimulates growth of MIA PaCa-2 cells through extracellular signal-regulated kinase phosphorylation. Oncol Lett 3: 307-310, 2012.

21. Bluemlein $\mathrm{K}$ and Ralser $\mathrm{M}$ : Monitoring protein expression in whole-cell extracts by targeted label- and standard-free LC-MS/MS. Nat Protoc 6: 859-869, 2011.

22. Zybailov B, Coleman MK, Florens L and Washburn MP: Correlation of relative abundance ratios derived from peptide ion chromatograms and spectrum counting for quantitative proteomic analysis using stable isotope labeling. Anal Chem 77: 6218-6224, 2005.

23. de Hoon MJ, Imoto S, Nolan J and Miyano S: Open source clustering software. Bioinformatics 20: 1453-1454, 2004.

24. Saldanha AJ: Java Treeview - extensible visualization of microarray data. Bioinformatics 20: 3246-3248, 2004.

25. Cotoi CG, Khorsandi SE, Pleşea IE and Quaglia A: Wholegenome DASL gene expression profiling of hepatocellular carcinoma sub-populations isolated by laser microdissection on formalin-fixed and paraffin-embedded liver tissue samples. Rom J Morphol Embryol 53: 893-902, 2012.

26. Kotorashvili A, Ramnauth A, Liu C, et al: Effective DNA/RNA co-extraction for analysis of microRNAs, mRNAs, and genomic DNA from formalin-fixed paraffin-embedded specimens. PLoS One 7: e34683, 2012. 
27. Nakanishi Y, Shimizu T, Tsujino I, et al: Semi-nested real-time reverse transcription polymerase chain reaction methods for the successful quantitation of cytokeratin mRNA expression levels for the subtyping of non-small-cell lung carcinoma using paraffin-embedded and microdissected lung biopsy specimens. Acta Histochem Cytochem 46: 85-96, 2013.

28. Feldman AS, Banyard J, Wu CL, McDougal WS and Zetter BR: Cystatin B as a tissue and urinary biomarker of bladder cancer recurrence and disease progression. Clin Cancer Res 15: 1024-1031, 2009

29. Lee M-J, Yu G-R, Park S-H, et al: Identification of cystatin B as a potential serum marker in hepatocellular carcinoma. Clin Cancer Res 14: 1080-1089, 2008.

30. Rivenbark AG and Coleman WB: Epigenetic regulation of cystatins in cancer. Front Biosci (Landmark Ed) 14: 453-462, 2009.

31. Keppler D: Towards novel anti-cancer strategies based on cystatin function. Cancer Lett 235: 159-176, 2006.

32. Kos J and Lah TT: Cysteine proteinases and their endogenous inhibitors: Target proteins for prognosis, diagnosis and therapy in cancer (Review). Oncol Rep 5: 1349-1361, 1998.

33. Gashenko EA, Lebedeva VA, Brak IV, Tsykalenko EA, Vinokurova GV and Korolenko TA: Evaluation of serum procathepsin B, cystatin B and cystatin C as possible biomarkers of ovarian cancer. Int J Circumpolar Health 72: 21215, 2013.

34. Masuishi Y, Arakawa N, Kawasaki H, Miyagi E, Hirahata F and Hirano H: Wild-type p53 enhances annexin IV gene expression in ovarian clear cell adenocarcinoma. FEBS J 278: 1470-1483, 2011.
35. Kim A, Enomoto T, Serada S, et al: Enhanced expression of Annexin A4 in clear cell carcinoma of the ovary and its association with chemoresistance to carboplatin. Int J Cancer 125: 2316-2322, 2009

36. Mogami T, Yokota N, Asai-Sato M, et al: Annexin A4 is involved in proliferation, chemo-resistance and migration and invasion in ovarian clear cell adenocarcinoma cells. PLos One 8: e80359, 2013.

37. Kajihara H, Yamada Y, Kanayama S, et al: Clear cell carcinoma of the ovary: Potential pathogenic mechanisms (Review). Oncol Rep 23: 1193-1203, 2010.

38. Tsuchiya A, Sakamoto M, Yasuda J, et al: Expression profiling in ovarian clear cell carcinoma: identification of hepatocyte nuclear factor-1 beta as a molecular marker and a possible molecular target for therapy of ovarian clear cell carcinoma. Am J Pathol 163: 2503-2512, 2003.

39. Duncan R, Carpenter B, Main LC, Telfer C and Murray GI: Characterisation and protein expression profiling of annexins in colorectal cancer. Br J Cancer 98: 426-433, 2008.

40. Lin L-L, Huang H-C and Juan H-F: Revealing the molecular mechanism of gastric cancer marker annexin A4 in cancer cell proliferation using exon arrays. PLoS One 7: e44615, 2012.

41. Zimmermann U, Balabanov S, Giebel J, et al: Increased expression and altered location of annexin IV in renal clear cell carcinoma: a possible role in tumour dissemination. Cancer Lett 209: 111-118, 2004 